\title{
Contrast Matching of Line Gratings obtained with NXE3XXX and EUV- Interference Lithography
}

\author{
Zuhal Tasdemir ${ }^{\mathrm{a}}$, Iacopo Mochi ${ }^{\mathrm{a}}$, Karen Garrido Olvera ${ }^{\mathrm{b}}$, Marieke Meeuwissen ${ }^{\mathrm{b}}$, \\ Oktay Yildirim ${ }^{\mathrm{b}}$, Rolf Custers ${ }^{\mathrm{b}}$, Rik Hoefnagels ${ }^{\mathrm{b}}$, Gijsbert Rispens ${ }^{\mathrm{b}}$, Roberto \\ Fallica $^{\mathrm{a}}$, Michaela Vockenhuber ${ }^{\mathrm{a}}$, and Yasin Ekinci ${ }^{\mathrm{a}}$ \\ a Laboratory for Micro- and Nanotechnology, Paul Scherrer Institute, CH-5232 \\ Villigen PSI, Switzerland \\ bASML Netherlands B.V., De Run 6501, 5504 DR Veldhoven, The Netherlands
}

\begin{abstract}
Extreme UV lithography (EUVL) has gained considerable attention for several decades as a potential technology for the semiconductor industry and it is now close to being adopted in highvolume manufacturing. At Paul Scherrer Institute (PSI), we have focused our attention on EUV resist performance issues by testing available high-performance EUV resists in the framework of a joint collaboration with ASML. For this purpose, we use the grating-based EUV-IL setup installed at the Swiss Light Source (SLS) at PSI, in which a coherent beam with $13.5 \mathrm{~nm}$ wavelength is used to produce a periodic aerial image with virtually $100 \%$ contrast and large depth of focus. Interference lithography is a relatively simple technique and it does not require many optical components, therefore the unintended flare is minimized and the aerial image is well-defined sinusoidal pattern. For the collaborative work between PSI and ASML, exposures are being performed on the EUV-IL exposure tool at PSI. For better quantitative comparison to the NXE scanner results, it is targeted to determine the actual NILS of the EUV-IL exposure tool at PSI. Ultimately, any resist-related metrology must be aligned and compared with the performance of EUV scanners. Moreover, EUV-IL is a powerful method for evaluating the resist performance and a resist which performs well with EUV-IL, shows, in general, also good performance with NXE scanners. However, a quantitative prediction of the performance based on EUV-IL measurements has not been possible due to the differences in aerial image formation. In this work, we aim to study the performance of EUV resists with different aerial images. For this purpose, after the real interference pattern exposure, we overlay a flat field exposure to emulate different levels of contrast. Finally, the results are compared with data obtained from EUV scanner. This study will enable not only match the data obtained from EUV- IL at PSI with the performance of NXE scanners, but also a better understanding of resist fundamentals by studying the effects of the aerial image on resist performance by changing the aerial image contrast in a controlled manner using EUV-IL.
\end{abstract}

Keywords: EUV lithography, image contrast, interference lithography, aerial image, high resolution patterning, photoresist, NXE3XXX

Further author information: (Send correspondence to Zuhal Tasdemir)

E-mail: zuhal.tasdemir@psi.ch, Telephone: +41563105807

International Conference on Extreme Ultraviolet Lithography 2017, edited by Paolo A. Gargini,

Patrick P. Naulleau, Kurt G. Ronse, Toshiro Itani, Proc. of SPIE Vol. 10450, 104501T

(C) 2017 SPIE · CCC code: $0277-786 X / 17 / \$ 18 \cdot$ doi: $10.1117 / 12.2280541$

Proc. of SPIE Vol. $10450104501 \mathrm{~T}-1$

Downloaded From: https://www.spiedigitallibrary.org/conference-proceedings-of-spie on 19 Apr 2019 Terms of Use: https://www.spiedigitallibrary.org/terms-of-use 


\section{INTRODUCTION}

As required by Moore's Law, the feature sizes in electronic devices have continued to decrease for the past several decades. Currently, the smallest feature size of $20 \mathrm{~nm}$ is used as the gate length of a logic device, and it is expected to be $12 \mathrm{~nm}$ in the next five years. ${ }^{1}$ The lithography process used to create semiconductor devices is at the center of the research efforts to decrease the transistors size. Nowadays, the industry is utilizing $193 \mathrm{~nm}-\mathrm{DUV}$ immersion lithography which has high throughput and is an established technology. Since this method is about to reach its resolution limit, EUV lithography using 13.5-nm-wavelength is the most likely successor of DUV. EUV resist materials are a key component of the EUV lithography process and have a major impact on its throughput and yield.

At Paul Scherrer Institute (PSI), we operate an interference lithography (IL) tool that is a powerful platform to perform early research on these materials. ${ }^{2,3}$ With EUV-IL, it is possible to perform an assessment of the performance of the most advanced resist material by probing their maximum resolution limit, but to obtain more quantitative results we need to perform a cross calibration of our data with the results obtained from a state-of-the-art scanner. In this paper, we developed a method to calibrate our EUV-IL exposure tool in order to match the contrast levels in ASML NXE scanners. Line/space patterns are exposed with both PSI's EUV-IL tool and ASML's NXE scanner and the results are compared. In the Section 2, we will introduce a brief background on the EUV- IL and our EUV-IL beamline at the SLS and then give the theoretical description of the sinusoidal aerial image of the EUV-IL tool and it will be compared with the aerial image of ASML NXE scanner. Experimental details, results and discussions are presented in the following sections.

\section{EUV INTERFERENCE LITHOGRAPHY}

While EUV technology goes through further research and development to prepare it for industrial use, resist companies have been developing and testing their resists to be used in EUVL for high-volume-manufacturing. The XIL-II beamline at the Swiss Light Source (SLS) is a dedicated beamline for EUV-IL for high-resolution patterning ${ }^{4}$ and it has demonstrated patterning capabilities down to $6 \mathrm{~nm}$ half-pitch (HP). ${ }^{5}$ In the past decades EUV-IL is successfully used for evaluation of EUV resists. ${ }^{6}$

\subsection{Aerial Image Formation}

In a lithographic tool aerial image formed on the wafer level is never ideal and suffers from some contrast loss due to the optics, illumination, and mechanical instabilities. Upon absorption, processing, and chemical reactions in resist, further contrast loss will take place where the aerial image is transformed to latent image. Most of the resist-induced contrast loss comes from the fact of acid diffusion during post-exposure baking. ${ }^{7}$ In a scanner, the aerial image of the reticle is produced by a complex optical system. One of the most useful metrics to evaluate the quality of the aerial image is the Normalized Image Log Slope (NILS). The NILS of an aerial image generated by a scanner depends on many factors, including the mask, the pattern geometry, the illumination and the residual flare and aberrations of the scanner optical system. In IL aerial image is generated in a different way, where the wafer is exposed to the interference fringe pattern generated by two plane waves. In our case, the two interfering waves are produced by a mask with two diffraction gratings as shown in Fig. 1. Let us assume to have a plane wave with wavelength incident on a mask with a grating with pitch $a$. The first-diffraction order is deflected by an angle that satisfies the grating equation:

$$
a \sin \theta=n \lambda
$$



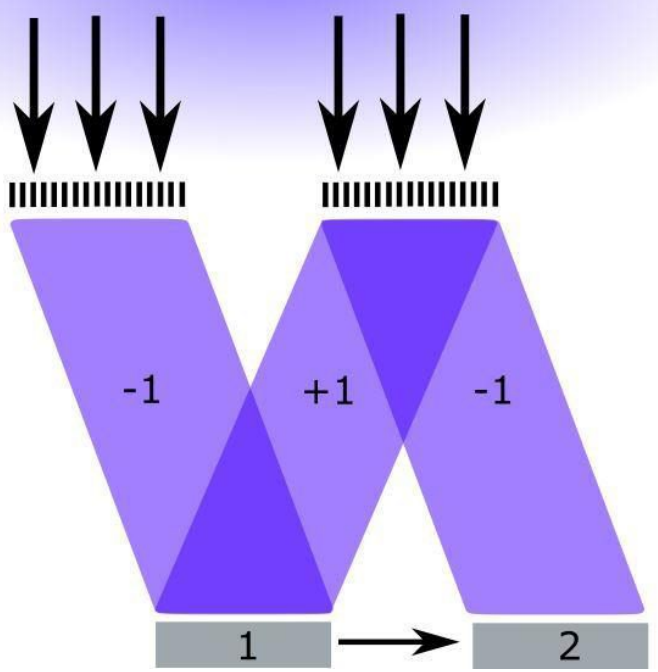

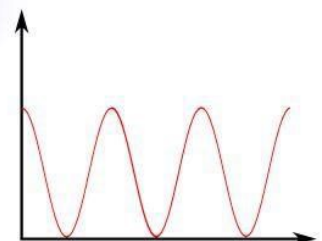

1. Modulation by EUV-IL

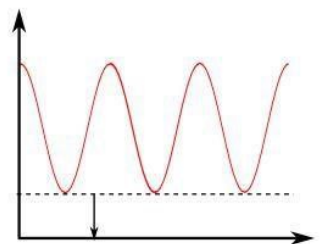

2. Artificial flare formation

Figure 1. The method used to create artificial flare level in EUV-IL. An interference pattern is exposed with converging first order diffraction beams from two gratings. Subsequently, the wafer is moved and a second exposure is performed with the diverging first order diffraction beam of the one of the gratings. In this figure, diffracting beams other than first-order are not shown for the sake of simplicity.

The two beams shown in Fig. 1 correspond to the $n=1$ and $n=-1$ diffraction orders from the two gratings and their phase difference in the interference plane is expressed by:

$$
\phi(x)=\frac{4 \pi \sin \theta}{\lambda}=\frac{4 \pi x}{a}
$$

This linear phase modulation does not depend on the wavelength allowing us to exploit the full bandwidth of our beam (about 4\%). Let $\mathrm{I}_{1}$ be the intensity of each of the first order diffraction beams. In the interference plane, the intensity distribution assumes a sinusoidal shape:

$$
I(x)=2 I_{1}\left(1+\sin \left(\frac{4 \pi x}{a}+b\right)\right)
$$

The interference image generated this way should have $100 \%$ contrast; in practice, imperfections in the gratings and aberrations in the incident beam may introduce a contrast loss that is accounted for introducing the flare term $b$ in Equation 3. The goal of this paper is to adjust our aerial image to match the contrast or the NILS of the aerial image generated by a scanner. For this reason, we performed a double exposure to add a custom flare level. This is done by translating the exposed wafer under one of the non-interfering diffracted beams and exposing it further, as shown in Fig. 1. If we let $D$ be the total desired exposure dose, to simulate the effect of a custom flare level $F$, we expose wafer to the interference pattern for a time $\mathrm{T}_{1}$ that corresponds to a dose $\mathrm{D}_{1}=\mathrm{D}-\mathrm{F}$. Then we expose the wafer to the non-interfering beam for a time $\mathrm{T}_{2}$ given by:

$$
T_{2}=4 T_{1} \frac{F}{D}
$$

If $I_{f}$ is the flare intensity, we can express the NILS of our aerial image as:

$$
N I L S=\left.0.25 a \frac{2 I_{1} \cos \left(\frac{4 \pi x}{a}\right) \frac{4 \pi}{a}}{2 I_{1}\left(1+\sin \left(\frac{4 \pi x}{a}\right)\right)+I_{f}}\right|_{x=0}=\frac{2 I_{1} \pi}{2 I_{1}+I_{f}}
$$


We can use this technique to tweak the NILS of our aerial image in a controlled way. If we assume a sinusoidal aerial image profile, we can calculate the NILS analytically. Let M be the image modulation and B the background level as shown in Fig. 2. The NILS is simply expressed by Equation 6 below.

$$
N I L S=\frac{M}{M+2 B} \pi=\mu \pi
$$

If we consider the $\mu$ between 0 and 1, the NILS values for EUV-IL beamline exposure tool could be listed as in Table 1. In the ideal conditions, which has zero background, $\mathrm{M}=1$ and $\mathrm{B}=0$, NILS has the maximum value of $\pi$. However, adding flare levels into the aerial image, the modulation decreases and the NILS value decreases. In this work, we increased flare levels incrementally from $0 \%$ to $30 \%$ during EUV exposure, as in the Table 1. The goal is to find the matching flare level in order to get the same NILS value in ASML scanner and EUV-IL exposure samples. The simulated aerial image of ASML NXE scanner is shown in Fig. 2 and the calculated NILS values are given in Table 2 .

Table 1. NILS Values for EUV-IL with different flare levels.

\begin{tabular}{ccc}
\hline \hline Flare Levels & NILS Value in $\pi$ & NILS Value \\
\hline \hline $0 \%$ & $1.00 \pi$ & 3.14 \\
\hline $5 \%$ & $0.90 \pi$ & 2.83 \\
\hline $10 \%$ & $0.82 \pi$ & 2.58 \\
\hline $15 \%$ & $0.74 \pi$ & 2.33 \\
\hline $20 \%$ & $0.67 \pi$ & 2.10 \\
\hline $30 \%$ & $0.54 \pi$ & 1.70 \\
\hline \hline
\end{tabular}

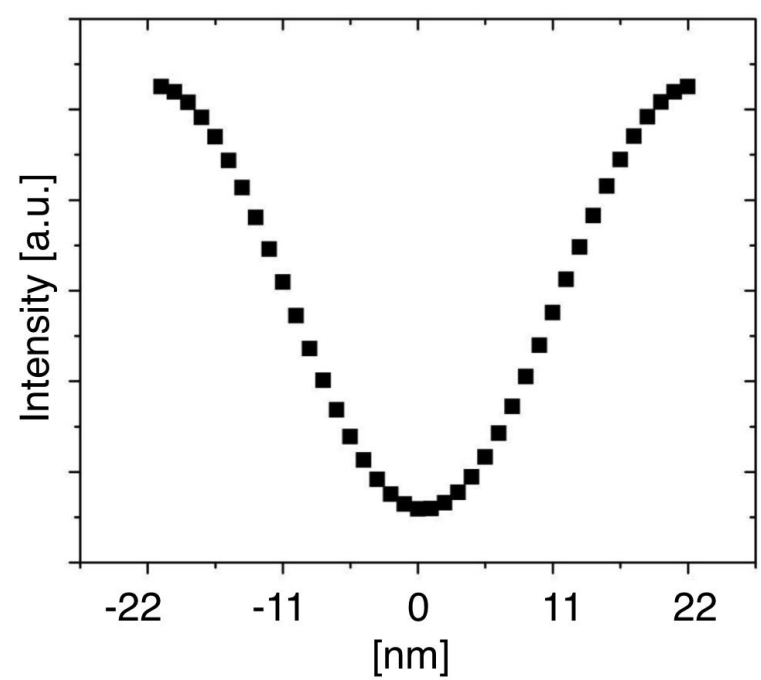

Figure 2. The simulated aerial image of ASML NXE scanner for the half-pitch of $22 \mathrm{~nm}$. 
Table 2. NILS Values for ASML Scanner, calculated from the experimental/simulated aerial images (e.g. HP $22 \mathrm{~nm}$ of Fig. 2).

\begin{tabular}{cc}
\hline \hline Feature & NILS at BE/BF \\
\hline \hline HP 22 Line/Space Horizontal Illumination & 2.53 \\
\hline HP 22 Line/Space Vertical Illumination & 2.22 \\
\hline \hline
\end{tabular}

\section{EUV-IL EXPOSURES}

\subsection{Mask Design and Fabrication}

The gratings consisting of HSQ (Hydrogen silsesquioxane) lines are patterned by e-beam lithography on 100-nm-thick Silicon Nitride $\left(\mathrm{Si}_{3} \mathrm{~N}_{4}\right)$ membranes. The pattern of lines/spaces is obtained by exposing two gratings with the equal period leading to sinusoidal aerial image. The mask is designed in a range of lines having $200 \mathrm{~nm}$ to $64 \mathrm{~nm}$ periods, corresponding to $50 \mathrm{~nm}$ to $16 \mathrm{~nm}$ $\mathrm{HP}$ on the wafer, as shown in Fig. 3. The grating pairs are arranged in a way that only first-order diffraction overlaps and creates the interference pattern. As for the intentional flare formation, we move the sample stage by certain amount and form the diverging first order diffraction beam of the one of the gratings. The mask fabrication starts on free-standing 100-nm-thick $\mathrm{Si}_{3} \mathrm{~N}_{4}$ membranes with an area of $9 \mathrm{~mm}^{2}$ within a Si frame area of $81 \mathrm{~mm}^{2}$. The high resolution resist, HSQ, is spin coated and grating patterns are written by e-beam lithography tool (Vistec EBPG 5000 Plus). This step is followed by PMMA coating and again e-beam lithography of the photonstop layer definition. Chromium and gold metal layers are coated and put in acetone for the lift-off for the seed layer outside of the grating areas. Finally, Nickel is coated by electroplating for the photon-stop metal layer. Additional details are available elsewhere. ${ }^{8}$

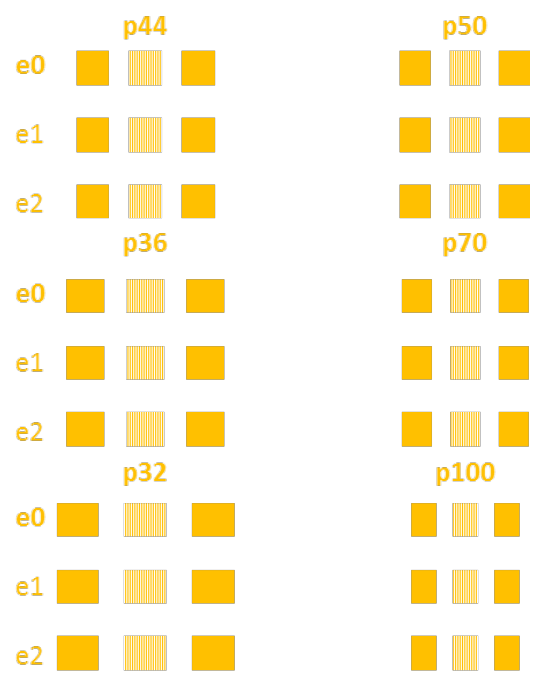

Figure 3. Design of a typical mask used in EUV-IL exposures at XIL beamline, SLS, PSI. The mask consists of grating pairs yielding line/space patterns of 22 to $50 \mathrm{~nm}$ HP between the grating pairs. Each HP field is repeated three times on the same mask. In a typical exposure, all the fields with different HPs are printed simultaneously. 


\subsection{Resist used and Process Parameters}

In this study of comparative IL and scanner exposures, we have used the same type of resist and the same process parameters for exposure and development. A standard chemically amplified resist (CAR) with a good performance at $22 \mathrm{~nm}$ line/space (LS) and standard EUV UL (underlayer) is used. Wafer processing is done in the class 1000 cleanroom at the XIL-beamline just before the EUV-IL exposure by using the parameters summarized in Table 3.

Table 3. Process Parameters for the resist used for EUV-IL exposures and analysis

\begin{tabular}{ccc}
\hline \hline Resist Name & Substrate & Development \\
\hline \hline Standard EUV CAR & Silicon 4" & MF26a for 25 s, DI-H2O rinse for 15 s \\
\hline \hline
\end{tabular}

\subsection{EUV Exposure Results and SEM Analysis}

EUV-IL exposures are performed on $4^{\prime \prime}$ silicon wafers starting from $0 \%$ intentional flare level within a predicted dose range of standard EUV CAR resist, then dose is adjusted for 5\%, 10\%, $15 \%, 20 \%$, and $30 \%$, respectively, in the same manner. The distance between the mask and the wafer surface is $750 \mu \mathrm{m}$ within a tolerance range of a few tens of micrometers. After the wafer development, the resulting lines/space patterns are inspected by scanning electron microscope (SEM) with an acceleration voltage of $1 \mathrm{kV}$ and a working distance of $3.0 \mathrm{~mm}$ (Supra V55, Zeiss, Germany). Afterwards, metrology analysis is done using a commercial software of SUMMIT, Lithometrix, USA. The results are shown in Fig. 4 as CD vs dose for different flare levels by EUV-IL and ASML NXE Scanner. As it is seen from the figure, when flare level increases the exposure latitude decreases and the slope of the lines (dose sensitivity) gets steeper. Without doing any dose calibration of EUV-IL tool, we could argue that ASML scanner data fits very well with the $10 \%$ flare level of EUV-IL exposure tool. The results will be discussed further in detail in Section 4.

\section{CALCULATION OF RESIST PARAMETERS}

In this section, some resist parameters are calculated in order to quantitatively compare the resist images produced by the two tools. Dose to clear and resist contrast, $\gamma$, parameters are then used to make a correlation between CD vs dose data for different flare levels of EUV-IL.

\subsection{Dose to clear}

For dose-to-clear measurement, open area flood exposures are done on $0.5 \mathrm{x} 0.5 \mathrm{~m}^{2}$ area of photoresist with EUV light at varying doses. After the steps of PEB and development processes, the thickness remaining in this open-frame area is measured for each dose. The thickness is normalized to the initial resist thickness. The thickness vs. dose curve is shown in Fig. 5.

\subsection{Resist Contrast}

The photoresist contrast is defined as the slope of the characteristic curve (normalized thickness vs dose) of the resist at the point of the dose to clear value. ${ }^{9,10}$

$$
\gamma= \pm\left.\frac{d T}{d \log _{10} D}\right|_{D=D t C}
$$




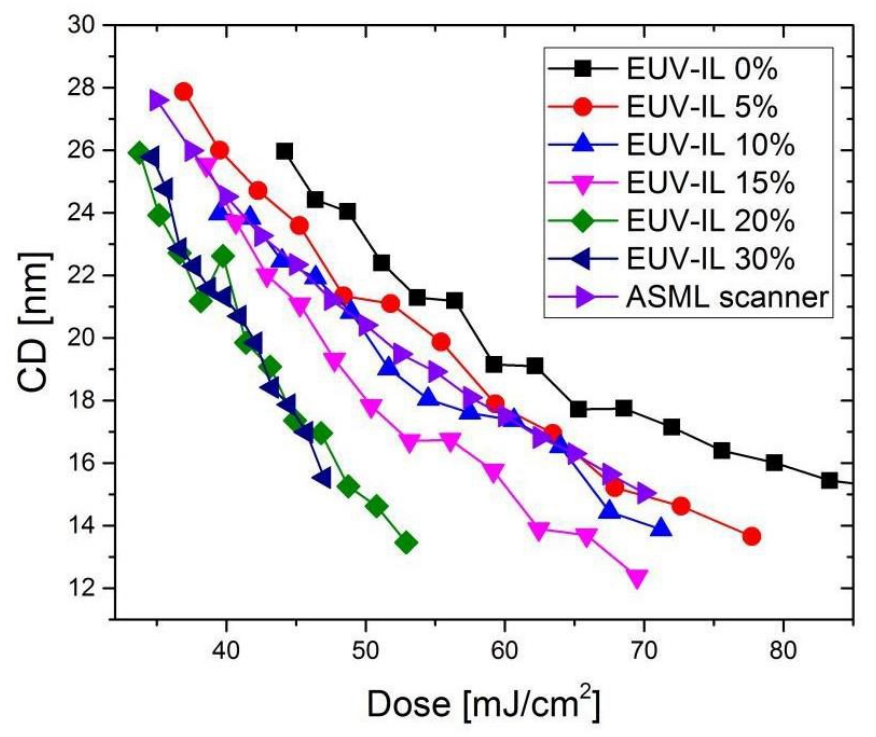

Figure 4. CD vs dose of HP $22 \mathrm{~nm}$ for different flare levels by EUV-IL and ASML NXE Scanner.

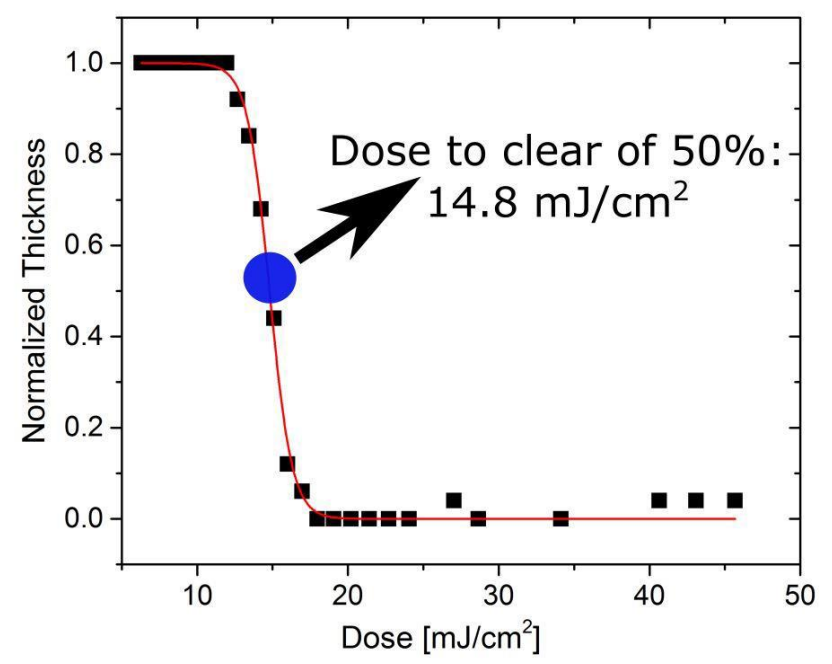

Figure 5. Normalized thickness vs dose curve after the development showing the dose to clear value at $50 \%$ of relative thickness.

Thus, where $T$ is the resist thickness removed during development, $D$ is the nominal exposure energy, and $D t C$ is the dose to clear value. The plus and negative signs are used in Equation 7 in order to keep the value of $\gamma$ positive. The calculated resist contrast value is found to be 11.4 as seen in Fig. 6 below, which is a resonable value for a state-of-the-art CAR. 


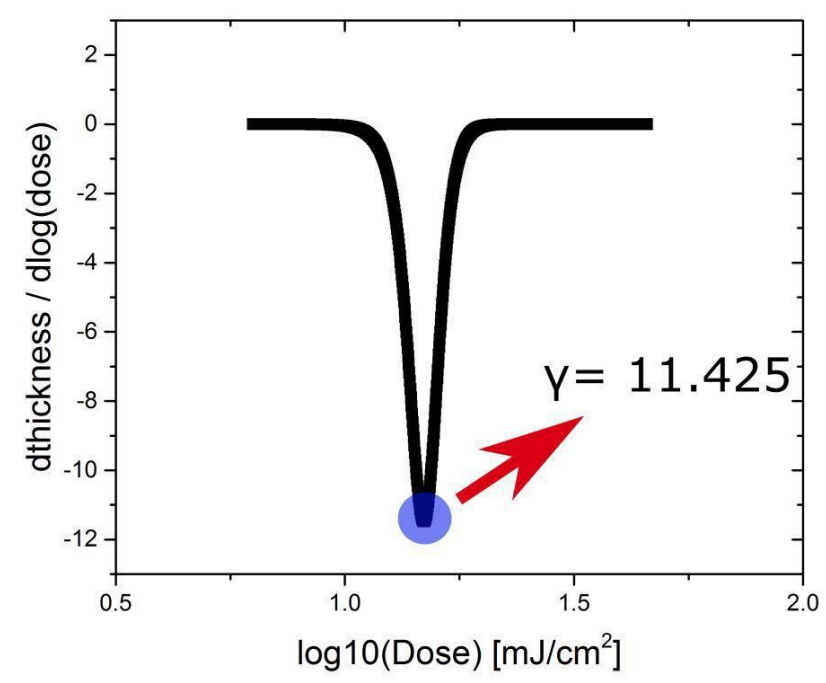

Figure 6. The calculated resist contrast, $\gamma$, value of 11.4 .

\subsection{NILS Value}

NILS is a useful metric of the aerial image quality and it is used to determine how optical parameters, such as aberrations of the optics, influence the image quality. When we consider a perfectly sinusoidal aerial image, and calculate the NILS simply by Equation 6, we get the maximum value of 3.14. The maximum NILS value assumes that there is no contrast loss due to the exposure tool. In Fig. 7, NILS vs Flare level is shown considering only the sinusoidal aerial image configuration.

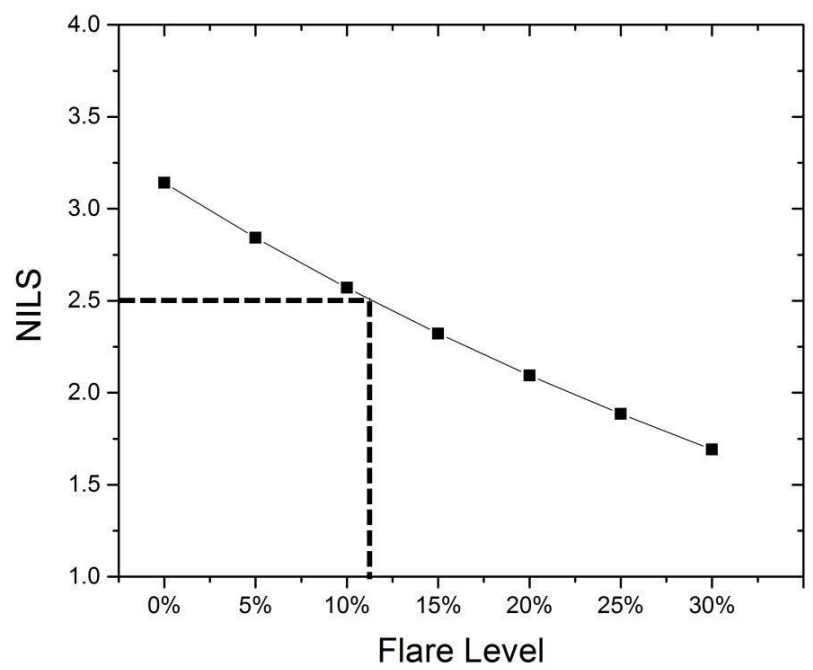

Figure 7. NILS vs Flare level for a sinusoidal aerial image showing around $11.5 \%$ flare level with NILS value of 2.5 . 


\section{DISCUSSION}

In order to compare the aerial images, i.e. flare levels of the data obtained from two different exposure tools, we have compared the dose-to-size for half-pitch of $22 \mathrm{~nm}$ of both data, as shown in Fig. 8. In this figure, NILS is varied over a wide range in EUV-IL, from a maximum value of 3.14 down to 1.69. As for the NXE scanner, NILS is 2.5 for half pitch of $22 \mathrm{~nm}$ as seen in Table 2 above. It is seen from the figure, we experimentally found that the dose to size increases with NILS. Our measurements show that the NILS of the aerial image of the NXE scanner corresponds approximately to the NILS value of EUV-IL having a flare level of $10 \%$ whereas according to the calculations the matching value should be $11.5 \%$. Therefore, the results of scanner and IL agree very well at this given half-pitch and for the present EUV resist. This result clearly demonstrates that EUV resist show the same performance with two very different tools and if the aerial images are matched.

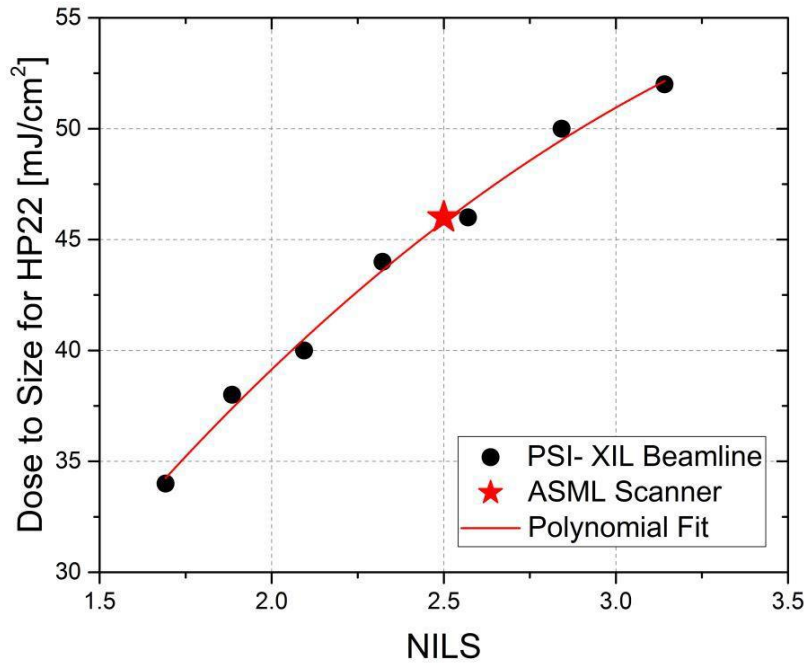

Figure 8. Comparison of Dose to Size for half pitch 22nm vs NILS value for both EUV-IL and NXE Scanner.

\section{CONCLUSIONS}

A method is developed to match the aerial image of two different lithography tools, i.e. PSI's EUV-IL and ASML's NXE scanner. The method is applied to EUV- IL by these two following steps : 1. using the first-order diffraction to create an interference pattern, and 2. another exposure is performed with the diverging first-order diffraction beam of one of the gratings. With this method, we were able to vary the NILS value of the aerial image without changing other factors such as half-pitch. The resulting line/space patterns are inspected by SEM with topview configuration and quantitative analysis of the lines is carried out with an image processing software. The measured CD vs dose curves show that ASML's NXE scanner data matches with about $10 \%$ flare level obtained by EUV-IL tool at PSI, resulting in a theoretically same NILS value for both tools. Since both tools have the same kind of aerial image at the given half-pitch, which is sinusoidal, NILS values obtained from two different techniques are calculated in the same way and compared with each other. These results are very encouraging in terms of showing the similar performance of resist with two different tools and very different methods of aerial image 
creation. We note that this $10 \%$ flare is the artificially added value into EUV-IL tool during exposure as described in Section 1. We note that this does not correspond to the same flare level of the same aerial image in the ASML scanner but corresponds to the same NILS value. The corresponding NILS value of the scanner is not only due to the flare but mainly it is due to aberrations in the optics, illumination, and the mask.

In the future, we would like to extend this study to other half-pitches and other resists such as inorganic resists, e.g. HSQ. We would like to develop a method which can reliably predict the resist performance, i.e. dose-to-size, line edge roughness, and exposure latitude, for a given NILS. A method with such a predictive power will enable assessing the performance of a resist without testing it with the scanner. The presented method enables the resist suppliers not only to test the EUV materials under ideal conditions but also offers a method to predict the performance with the scanner with different NILS values dependent on the illumination conditions. In particular, it is of great importance to estimate the extendibility of the resists for high-NA EUV scanners for future technology nodes where the aerial image is not available yet but can be calculated.

\section{REFERENCES}

[1] ITRS, "International technology roadmap for semiconductors 2015 report." ITRS, 2015 Edition (2016).

[2] Buitrago, E., Nagahara, S., Yildirim, O., Nakagawa, H., Tagawa, S., Meeuwissen, M., Nagai, T., Naruoka, T., Verspaget, C., Hoefnagels, R., Rispens, G., Shiraishi, G., Terashita, Y., Minekawa, Y., Yoshihara, K., Oshima, A., Vockenhuber, M., and Ekinci, Y., "Sensitivity enhancement of chemically amplified resists and performance study using extreme ultraviolet interference lithography," J. Micro/ Nanolith. MEMS MOEMS 15 (2016).

[3] Passarelli, J., Murphy, M., Re, R. D., Sortland, M., Hotalen, J., Dousharm, L., Fallica, R., Ekinci, Y., Neisser, M., Freedman, A., and Brainard, R., "Organometallic carboxylate resists for extreme ultraviolet with high sensitivity," J. Micro/Nanolith. 14 (2015).

[4] Mojarad, N., Gobrecht, J., and Ekinci, Y., "Interference lithography at EUV and soft X-ray wavelengths: Principles, methods, and applications," Microelectron. Eng 143, 55-63 (2015).

[5] Fan, D. and Ekinci, Y., "Photolithography reaches $6 \mathrm{~nm}$ half-pitch using extreme ultraviolet light," J. Micro/Nanolith. MEMS MOEMS 15 (2016).

[6] Goethals, A., and F. Van Roey, R. G., Solak, H., and Ekinci, "Progress in EUV resist performance," J. Photopolym. Sci.Tech. 19 (2006).

[7] Bienert, M., Aksel, G., Natt, O., Lowisch, M., Grupner, P., Heil, T., Garreis, B., Schenauoen, K. V. I., and Hansen, S., "Imaging budgets for EUV optics: ready for 22nm node and beyond," Proc. SPIE Advanced Lithography, 72711B1B13 (2009).

[8] Buitrago, E., and D. Fan, R. F., Kulmala, T. S., Vockenhuber, M., and Ekinci, Y., "SnOx high-efficiency EUV interference lithography gratings towards the ultimate resolution in photolithography," Microelectronic Engineering 155, 44-49 (2016).

[9] Hurter, F. and Driffield, V., "Photochemical investigations and a new method of determination of the sensitiveness of photographic plates," J. Soc. Chem. Ind. 19 (1980).

[10] Mack, C., "Lithographic optimization using photoresist contrast," Microelectron. Manuf. Technol. 14 (1991). 